\title{
The FRAM Telescope at the Pierre Auger Observatory
}

\author{
Jiri Blazek ${ }^{* a}$ for the Pierre Auger Collaboration ${ }^{b}$ \\ ${ }^{a}$ Institute of Physics, Prague, Czech Republic \\ ${ }^{b}$ Observatorio Pierre Auger, Av. San Martín Norte 304, 5613 Malargüe, Argentina \\ E-mail: auger_spokespersons@fnal.gov \\ Full author list: http://www.auger.org/archive/authors_icrc_2017.html
}

\begin{abstract}
The knowledge of both the long-term characteristics of the atmosphere and of its immediate state is of vital importance for the study of extensive air showers. The F/Photometric Robotic Atmospheric Monitor (FRAM), installed at the Pierre Auger Observatory, is an autonomously operating instrument which measures the integral light extinction in a given direction using stellar photometry. Its primary purpose is the rapid observation of atmospheric conditions soon after the fluorescence detectors of the Observatory have recorded a particularly interesting shower. This information is crucial for the study of showers with anomalous longitudinal profiles, which are predicted by hadronic interaction models but whose observation can also arise as a consequence of the presence of clouds or aerosol layers. The current status of the analysis is presented. In addition to the primary function of the FRAM, the integral aerosol content can be obtained by utilizing the large number of stars identified in the wide-field images of the instrument. The method for this is also briefly described.
\end{abstract}

35th International Cosmic Ray Conference

10-20 July, 2017

Bexco, Busan, Korea

${ }^{*}$ Speaker. 


\section{The Pierre Auger Observatory}

The Pierre Auger Observatory utilizes a hybrid design for the simultaneous detection of cosmic rays of ultrahigh energy, making use of both the surface and the fluorescence detectors [1]. The surface detector (SD) array comprised of water-Cherenkov stations observes a portion of the incoming shower hitting the ground. It functions with a full uptime of 24 hours per day and covers an area of $3000 \mathrm{~km}^{2}$ in a triangular grid with a spacing of $1.5 \mathrm{~km}$. The arrival direction and the energy of the primary particle can be inferred from the particle densities recorded by the SD stations. The fluorescence detector (FD) concurrently views the fluorescence light emitted during the development of the shower in the atmosphere. Its telescopes are concentrated at four sites, with a combined field of view of $180^{\circ}$ per site, overlooking the SD array. The FD is capable of measurement only during nights with a low moon fraction and sufficiently clear weather, resulting in a duty cycle of approximately $15 \%$. By observing the longitudinal profile of the shower the detector is able to perform an almost calorimetric measurement, where the energy corresponding mostly to the electromagnetic part of the shower cascade is obtained by integrating the fitted fluorescence profile.

The collected fluorescence light is attenuated by molecular scattering and by scattering on aerosols present between the detector position and the shower. The estimation of the energy of the shower thus depends strongly on the state of the atmosphere. The presence of absorbing or scattering layers can further cause the observation of additional features in the profile which have an origin outside of hadronic interactions.

Precise knowledge of the atmospheric conditions is thus of major importance. The Pierre Auger Observatory employs an extensive atmospheric monitoring program; see [1] for the description of the various instruments. The respective installations record the atmospheric observables at regular, periodic intervals. However, in order to study particularly interesting and rare showers, a greater spatial and temporal resolution is crucial. In the following we will describe the Shoot-theShower (StS) program and in particular the F/photometric Rapid Atmospheric Monitor (the FRAM telescope), which were designed to rapidly perform a measurement after a shower was recorded by the fluorescence detector.

\section{Shoot-the-Shower: the FRAM Telescope}

The instrument consists of a wide-field camera, optical focuser and a set of rotating filters, jointly attached to a remotely controlled mount. This system is encased in a dome with dimensions of approximately $2.5 \mathrm{~m} \times 2.5 \mathrm{~m}$. The data acquisition and the operation of the hydraulics of the enclosure are handled by a control computer. The remotely operated camera features a field of view of $7^{\circ} \times 7^{\circ}$. Using a 30 second exposure, thousands of stars are typically seen in a single image, depending on the particular area in the sky. The instrument is located at the Los Leones site, very near the fluorescence detectors. Fig. 1a shows the telescope with the enclosure in an open state.

The Shoot-the-Shower analysis chain functions as follows. The raw data recorded by the surface and fluorescence detectors are being passed to a centrally located computer. A hybrid reconstruction is performed and the retrieved parameters of the shower are passed further to a control computer of the FRAM telescope. A set of predefined triggers is applied: currently, these 
look for showers with an anomalous profile, highly energetic showers and candidates for a shower caused by a highly energetic gamma primary. If the cuts are passed, the telescope records images along the reconstructed axis of the shower. The retrieval of the data from the SD and FD and the subsequent reconstruction takes approximately 3 to 5 minutes, after which the telescope is ready to shoot the first picture. The median number of recorded images per trigger is 5 , but the number of recorded images could also be significantly higher in case of inclined shower geometries coming parallel to the focal plane of the FD, spanning a wide range in azimuth angle (Fig. 1b). Events with with fewer than 4 images are discarded, as in many cases they don't cover a sufficient portion of the development of a shower as recorded by the FD.

During analysis, stars are extracted from the images and then matched with sources in the Tycho2 [2] star catalog. Comparing the light fluxes obtained using star photometry on the images recorded by FRAM with the values listed in Tycho2, an extinction for each source can be retrieved. A global fit can then be performed based on the predicted dependence of the extinction on the airmass. Any significant variations from the fit then point to the presence of attenuating inhomogeneities. The most frequently triggered type of event is the double-bump candidate, described in the following section.

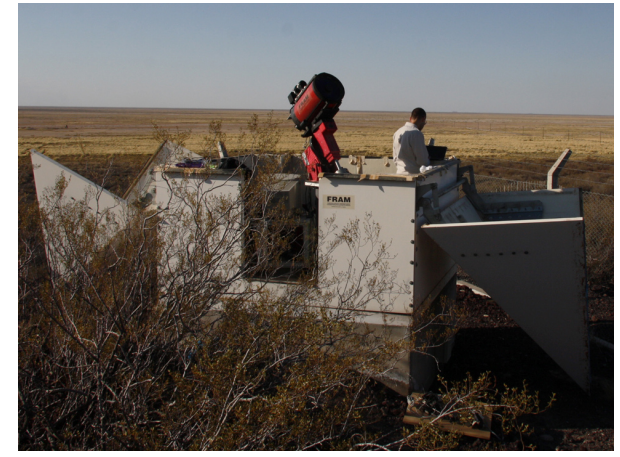

(a)

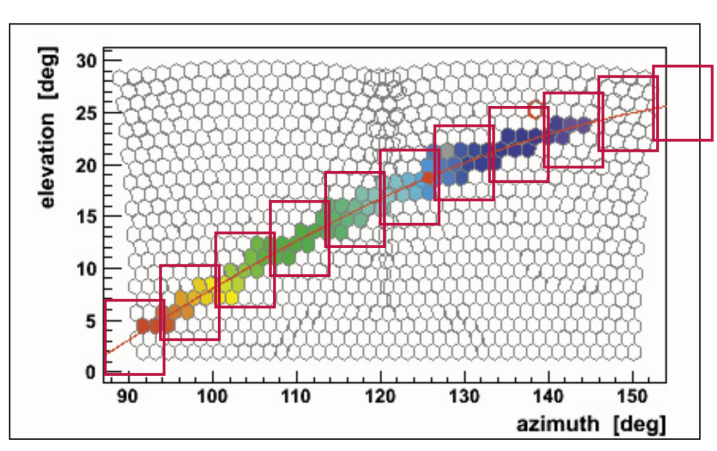

(b)

Figure 1: a) A photo of the FRAM telescope, with an open enclosure. b) An illustrative sketch of the area covered by FRAM for a single event, in this case recorded by two neighboring fluorescence telescopes at a single site.

\section{Double-Bumps}

The showers with an anomalous longitudinal profile, or the so-called double-bump showers, differ markedly from a universal profile described, e.g., by the empirical Gaisser-Hillas function [3]. An example of such a shower simulated in a Monte Carlo generator is shown in the left part of Fig. 2. To be classified as anomalous, the profile should feature a prominent second maximum or a clearly distinguishable set of two additional inflexion points. Such a property can be explained by a particle originating in the first several generations of a shower development which receives a sufficiently high fraction of the energy of the primary particle. If it then propagates deeply into the atmosphere without losing a significant portion of its energy, it creates a secondary sub-shower which can be clearly distinguished from the products of the primary cascade. Such a 
process however requires a light nucleus as the primary particle, since the nucleons in heavy nuclei do not possess sufficient energies and the resulting sub-shower would be too insignificant for detection and would get smeared out among the sub-showers originating from the rest of the nucleons. Observing an anomalous shower profile at a given energy would then represent direct evidence of the presence of a light fraction in the mass composition of the primary cosmic rays. Furthermore, given that a sufficient dataset is acquired, the distribution of the profile maxima of the secondary sub-showers can be used to inspect the particle-air cross section.

Several studies [4], [5] were performed on very large samples of Monte Carlo generated events, using various definitions of anomalous profiles for the purposes of their identification. The concrete quantitative predictions of the rate of double bumps depend on the particular definition used, on the energy of the primary particle, on the hadronic interaction model employed in the Monte Carlo simulations and on the type of the primary particle. For a proton primary, the ratio of anomalous profiles $N_{\text {anom }}$ to the total number of events $N_{\text {tot }}$ is of the order of $10^{-3}$, see the right part of Fig. 2. A very reliable rejection of false positives is thus of crucial importance. Studying data from the fluorescence detector of the Pierre Auger Observatory, it is clear that the overwhelming majority of profiles displaying anomalous features is the result of atmospheric conditions in the vicinity of the shower axis. There are two mechanisms through which a shower with a regular longitudinal profile could masquerade as anomalous. Either there is a cloud in between the axis and the detector, obfuscating part of the fluorescence light and creating an artificial minimum, or the cone of Cherenkov light, narrowly collimated along the shower axis, hits a cloud or an aerosol layer and scatters a portion of the light towards the fluorescence detector, creating an artificial maximum in the profile. The FRAM telescope is sensitive to clouds between the telescope and the edge of the atmosphere and is able to identify both types of events. The exception is the case where the perpetrator is a uniformly distributed, thin homogeneous layer. Such an extended layer can however be recognized by the other atmospheric monitoring devices.
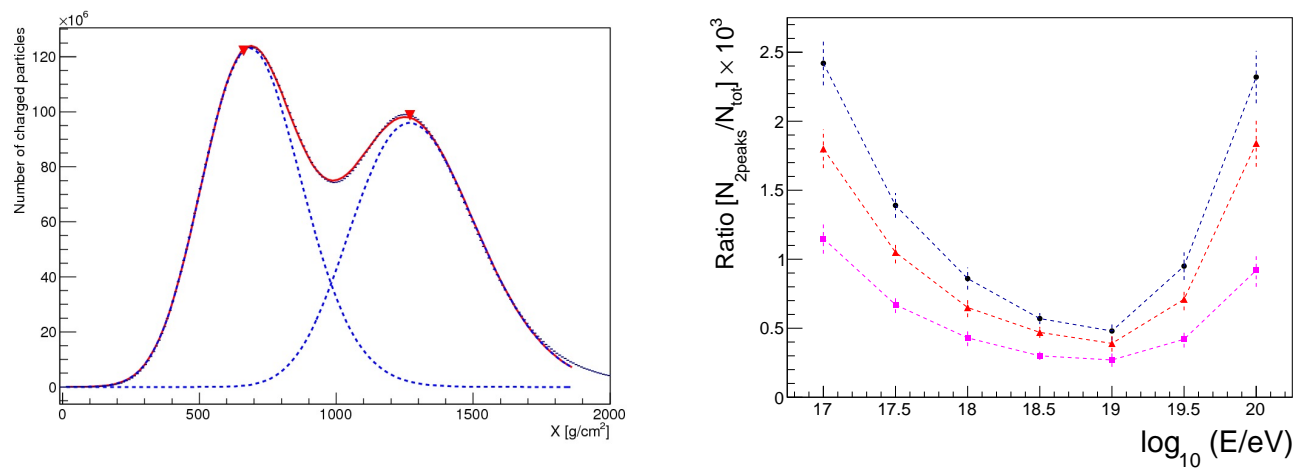

Figure 2: Left: a sample example of an anomalous longitudinal profile, generated by the CONEX software for a proton primary with energy $10^{20} \mathrm{eV}$. Right: dependence of the ratio of double bumps on energy for a proton primary. The hadronic interaction model EPOS-LHC was used. The three lines represent three reasonable choices for a parameter defining an anomalous profile. Taken from [5]. 


\section{Image Processing}

Each telescope of the fluorescence detector has a field of view of $30^{\circ} \times 30^{\circ}$ in azimuth and altitude, with a minimal angle of approximately $1.5^{\circ}$ above the horizon. Accordingly, the FRAM telescope is configured to cover a range in altitude from roughly $35^{\circ}$ all the way to the horizon, depending on the geometry of the particular shower. This range is more than sufficient, since the angular span of the FD profile is usually significantly smaller than $30^{\circ}$. Moreover, by scanning a larger portion of the sky than that covered by an air shower we can also make sure that a portion of the FD profile is not missing due to a presence of a cloud.

During analysis, the processed images have to be first calibrated by applying a correction with regards to "flat" and "dark" images. The process is described in detail in [6]. After removing a large part of the background, stars are then identified, their coordinates are extracted and their light flux is calculated by means of aperture photometry. Pairs of stars closer than twice the aperture are removed to avoid any light cross-contamination. Finally, the obtained fluxes are compared to values provided by the Tycho2 catalog [2] for stars that should be present in that particular spot in the night sky. The difference in magnitudes then gives the extinction for the respective star. By analyzing several wide-field images, hundreds to thousands of stars spanning a wide range in altitude (and the airmass, respectively) can be identified on a single scan and a fit can be performed to obtain the extinction coefficient. The predicted dependence on the airmass $A$ is simple:

$$
m_{\text {inst }}=m_{\text {cat }}+Z+k A+\text { correction terms }
$$

where $m_{\text {inst }}$ is the apparent light flux in magnitudes, $m_{\text {cat }}$ is the flux of a matched star in the Tycho2 catalog, $Z$ is the value of the zeropoint, which fixes the otherwise arbitrary scale and is the primary calibration constant and $k$ is the extinction coefficient. The last part of the equation refers to terms correcting various properties of the optical system and of the star catalog itself. The correction terms are fitted once for a large sample of scans featuring no cloud contamination and then kept fixed.

Finally, one obtains a plot of the extinction profile for a given scan, such as shown in the middle column of Fig. 3. The left column shows the associated FD profile, which triggered the scanning. The right column shows deviations from the fit plotted against slant depth, to be directly comparable to the FD profile. Fig. $3 b$ is an example of a very clear event, with the stars clustered along the predicted extinction curve and no signs of any clouds. The consequences of their presence could be seen in Fig. $3 \mathrm{~h}$ - many stars are attenuated by several magnitudes and their location differs from the extinction curve by many sigma. In this case the fitted curve is not reliable anymore, since the regions with attenuated stars are causing an artificial flattening of the slope. Note that, apart from a region near $8^{\circ}$ in altitude, the stars are still visible through the clouds. In many other cases whole portions of the profile are missing.

The presence of clouds is ascertained by a strict visual inspection. However some types of densely populated profiles could prove misleading and hide a slightly deviating population of stars. To get a better quantitative handle we inspect the difference between the measured and fitted extinction for each star and plot it against the slant depth (Fig. 3), so it can be directly compared to the FD profile (this step requires knowledge about the geometry of the shower). Negative values represent stars with missing light and point to the presence of a thin cloud. Positive values indicate 
an abundant light. This is caused mainly by the aforementioned flattening of the extinction curve, but simple statistical fluctuations and effects like possible inhomogeneities of the star catalog could also play a role.

The event in the middle row in Fig. 3 is a curious case: the FD profile features a clear secondary peak, whose width is unphysically narrow in the sense that it differs vastly from a universal shape of a shower created by a proton primary. The extinction profile in Fig. 3e does not exhibit significant deviations from the fit. However, Fig. 3f shows a clearly attenuated population in the vicinity of $1200 \mathrm{~g} / \mathrm{cm}^{2}$, which also exactly corresponds to the location of the maximum in Fig. 3d. Thus in this case the anomalous profile was a result of scattering of the Cherenkov light off a thin cloud.

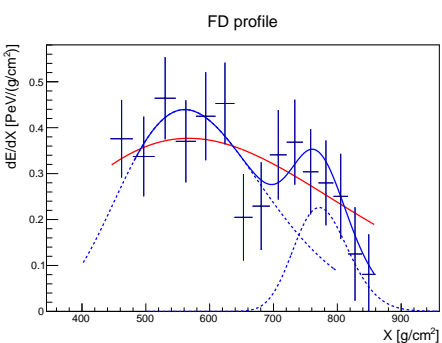

(a)

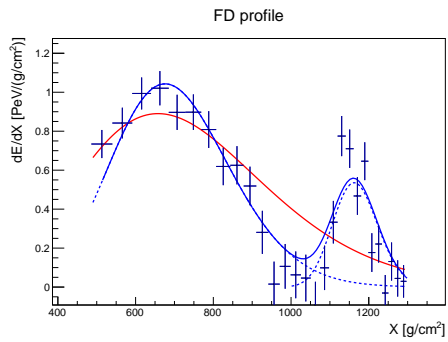

(d)

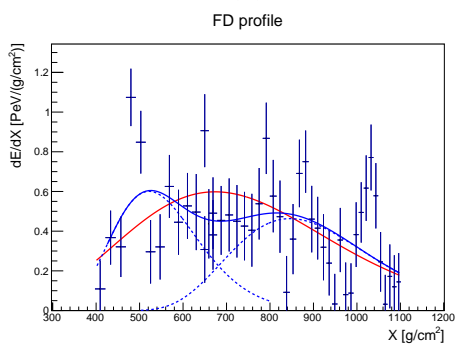

(g)

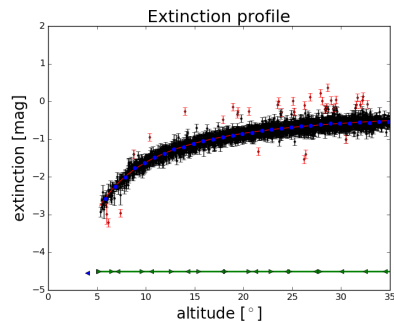

(b)

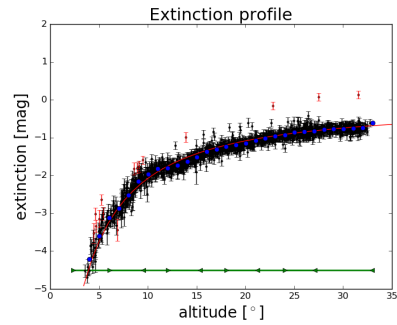

(e)

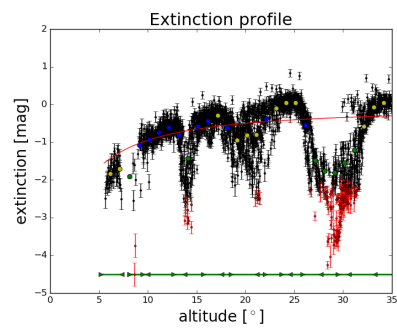

(h)

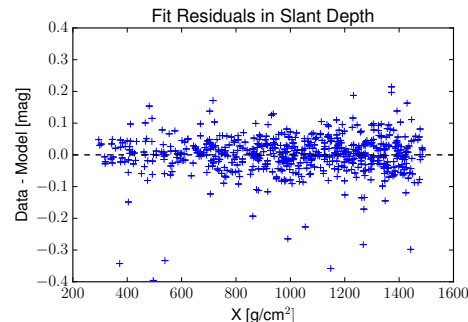

(c)

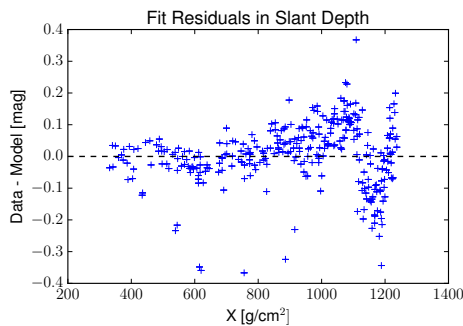

(f)

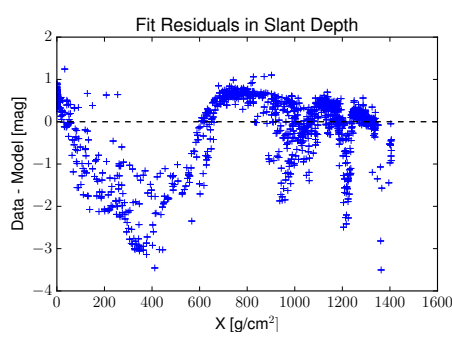

(i)

Figure 3: Every row represents a single shower and the associated FRAM scan. Left column: a fluorescence profile recorded by the FD which passed the double-bump trigger. The red line represents a fit of a regular Gaisser-Hillas function, blue line a combination of two G.-H. functions. Middle column: an extinction profile recorded by FRAM. The red line shows the fitted function 4.1. The green lines near the $\mathrm{x}$ axis plot the range in altitude for every image in the scan. Each point represents a single star, large outliers being plotted in red. Right column: the differences between the measured and the fitted extinction value for every star, recast in terms of the slant depth.

The presence of such inhomogeneities also generally leads to larger fluctuations in fit residuals of individual stars in the profile. Consequently, a new cut on the Root Mean Square (RMS) was 
introduced in order to eliminate events of this type. Its value was set to $0.1 \mathrm{mag}$ and its application resulted in a loss of approximately 5\% of events. An illustrative graph presenting the distribution of the RMS is shown in Fig. 4. Several large outliers were already excluded in order to make the graph clearer. The shown data sample consists of scans previously classified as clear from several months in 2016.

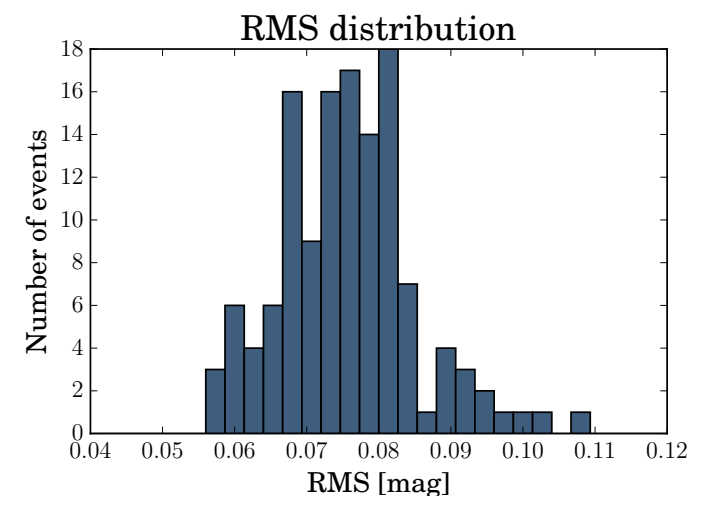

Figure 4: Average fluctuations from a predicted profile.

\section{Measuring the Aerosol Content of the Atmosphere}

As already mentioned in section 1, the attenuation of incoming fluorescence light attributable to aerosols plays an important role in the shower reconstruction and has a direct impact on the estimated energy of the primary particle. The FRAM telescope incidentally produces scans over a wide range of airmass while looking for clouds during the StS program. This can be utilized for the precise determination of the attenuation coefficient $k$, obtained by fitting the simple functional form 4.1 while carefully handling the contributions of the various correction terms. Moreover, the StS scans are recorded in the B filter (with effective midpoint wavelength of approximately 440 $\mathrm{nm}$ ), where the attenuation due to aerosols is much smaller than the contribution by molecular scattering. Because the optical system has a finite passband and Rayleigh scattering is also wavelength dependent, the two need to be attentively combined, corrected for the temporal fluctuations in air density using data from the GDAS network and then finally subtracted from $k$ to obtain the aerosol contribution. This process is described in detail in [6].

The method works well during cloudless nights with a low moon fraction, where no additional or missing light is distorting the quality of the fit. The RMS of the fit residuals is $\langle R M S\rangle=0.075$ mag. Their dependence on various parameters of the fit is shown in Fig. 5. The residuals are reasonably small, with no identifiable major data populations which systematically and significantly deviate. The statistical errors connected to the determination of the coefficient $k$ itself are of the order of 0.01 .

\section{Summary}

We have introduced the FRAM telescope and its role within the context of the Pierre Auger Observatory, in particular in the Shoot-the-Shower program. The described cloud identification 

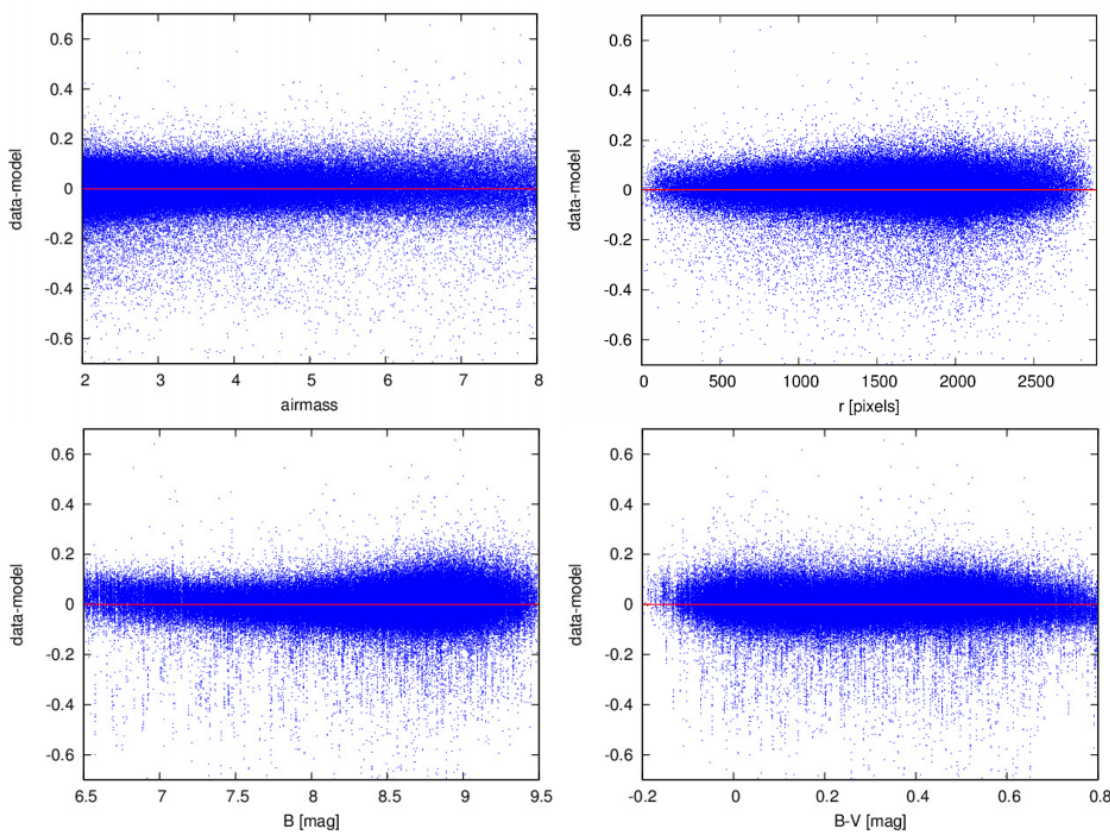

Figure 5: Residuals of the global fit as a function of the airmass, the distance from the center of the image, the magnitude measured in the B filter and the color dependence of the measurement in the B and V filters.

method based on stellar photometry performed on a set of wide-field images serves as a basis for the selection of showers featuring longitudinal profiles undistorted by atmospheric effects. The question of whether the obtained sample of candidate events is indeed showing anomalous bumpy features originating in hadronic interactions is still open and the work on the interpretation of the dataset is ongoing. We have also very briefly described a novel method for the measurement of attenuation due to aerosols using scans across a wide range of airmass. The statistical uncertainties of the obtained extinction coefficients are of the order of 0.01 and thus sufficiently low for the purposes of atmospheric monitoring, but the estimation of the various connected systematic errors is difficult and is still work in progress.

\section{References}

[1] A. Aab et al. [Pierre Auger Collaboration], Nucl. Instrum. Meth. A 798, 172 (2015), [arXiv:1502.01323 [astro-ph.IM]].

[2] E. Hog et al., Astron. Astrophys. 355, L27 (2000).

[3] T. K. Gaisser and A. M. Hillas, Proc. 15th ICRC 1977, Plovdiv, Bulgaria, 8, 353 (1977).

[4] C. Baus, R. Engel, T. Pierog, R. Ulrich and M. Unger, Proc. 32nd ICRC 2011, Beijing, China, 2, 206 (2011) [arXiv:1111.0504 [astro-ph.HE]].

[5] J. Blazek [Pierre Auger Collaboration], EPJ Web Conf. 144, 01009 (2017).

[6] J. Ebr et al. [Pierre Auger Collaboration], EPJ Web Conf. 144, 01011 (2017). 